\title{
Hidden Costs of Graphical User Interfaces: Failure to Make the Transition from Menus and Icon Toolbars to Keyboard Shortcuts
}

\author{
David M. Lane \\ H. Albert Napier \\ S. Camille Peres \\ Anikó Sándor \\ Department of Psychology \\ Rice University
}

\begin{abstract}
Graphical interfaces allow users to issue commands using pull-down menus, icon toolbars, and keyboard shortcuts. Menus and icon toolbars are easier to learn, whereas keyboard shortcuts are more efficient. It would seem natural for users to migrate from the use of easy-to-learn menu and icon methods to the more efficient method of keyboard shortcuts as they gain experience. To investigate the extent to which this transition takes place, 251 experienced users of Microsoft Word were given a questionnaire assessing their choice of methods for the most frequently occurring commands. Contrary to our expectations, most experienced users rarely used the efficient keyboard shortcuts, favoring the use of icon toolbars instead. A second study was done to verify that keyboard shortcuts are, indeed, the most efficient method. Six participants performed common commands using menu selection, icon toolbars, and keyboard shortcuts. The keyboard shortcuts were, as expected, the most efficient. We conclude that even experienced users are inefficient in their use of graphical interfaces. One possible way to improve user efficiency is for training programs to provide a roadmap for users to make the transition from using pull-down menus and clicking icon toolbars to issuing keyboard shortcuts.
\end{abstract}

\section{INTRODUCTION}

A good interface should be (a) easy for novices to learn, (b) efficient for experts to use, and (c) provide the means for users to make the transition from the easy-to-learn but inefficient methods of novices to the more difficult-to-learn and

The research for this article was partially funded by the Center on the Management of Information Technology at Rice University.

Requests for reprints should be sent to David M. Lane, Rice University, Department of Psychology-MS 25, P.O. Box 1892, Houston, TX 77251-1892. E-mail: lane@rice.edu 
efficient methods of experts $(\mathrm{Wu}, 2000)$. The graphical interface in common use today appears to meet these criteria fairly well. Several studies have found that software with graphical interfaces is easy to learn and use (Davis \& Bostrom, 1992; Galitz, 1996; Temple \& Sloan, 1990). One important factor contributing to the ease of use is that pull-down menus and icon bars make the set of possible commands visible during the use of the software, thus eliminating the need to memorize commands (Galitz, 1996).

The presence of easy-to-use features such as pull-down menus and icon bars does not preclude graphical interfaces from having advanced features such as macros, templates, stylesheets, and keyboard shortcuts. Thus graphical interfaces can be very efficient for expert users.

It is reasonable to suppose that, with experience, novice users of a software application would become efficient users, relying greatly on the use of advanced features. Although there is relatively little data available concerning such usage, there is reason to doubt that experienced users are necessarily efficient users. In a study examining the use of a text editing software (XEDIT), Rosson (1984) found that the use of more powerful commands did not increase as a function of experience and that even experienced users were not using the software efficiently. Similarly, Bhavnani and John (1997) found that users of computer-aided design (CAD) software did not typically use the most advanced and efficient methods.

This article focuses on one aspect of efficiency-the use of keyboard shortcuts rather than pull-down menus and icon bars. It would seem that learning keyboard shortcuts would be easier and more frequent than learning the more complex efficient methods for using XEDIT and CAD software. First, keyboard shortcuts, by their very nature, are not complex. Second, the graphical interface is well-suited for helping users to make the transition: (a) keyboard shortcuts and icons associated with a command are often shown when one chooses a command from a pull-down menu and (b) some programs such as Microsoft Word have an option to show the corresponding keyboard shortcut when users position the mouse over an icon on an icon toolbar. Thus, the graphical interface appears to provide strong support for the transition to keyboard shortcuts.

Does this apparent strong support for a transition to keyboard shortcut result in the frequent use of these shortcuts? Study 1 assesses the frequency with which keyboard shortcuts are used by administering questionnaires to experienced users of Microsoft Word concerning their preferred methods of issuing commands. Study 2 collects performance data to evaluate whether keyboard shortcuts are, in fact, more efficient than menus or icon toolbars.

\section{STUDY 1}

To determine methods used to execute commands in Microsoft Word, a survey was conducted asking participants what percentage of the time they used the menu, alternate menu, keyboard shortcut, and icon methods for issuing frequently used commands. For each command, they were asked to provide an estimate of the percentage of the time they used each method to issue the command. 


\subsection{Method}

The survey was distributed to 332 individuals working at energy companies, pipeline companies, the federal government, hospitals, and educational institutions as computer software instructors. These participants were asked to make estimates for the following commands: opening a document, creating a new document, saving a document, printing a document, copying information to the clipboard, removing (cutting) information from a document, pasting the content of the clipboard to a document, undoing the last operation, repeating the last operation, finding a particular sequence of characters, checking the spelling in a document, bolding a selected set of characters, underlining a set of selected characters, italicizing a set of selected characters, and changing the name of a file. For each command they were asked the percentage of time they used each of the various method for issuing the command. One such question is shown in Figure 1.

Demographics. A total of 251 of the 332 surveys distributed were returned, representing a return rate of $76 \%$. Most of the participants were professionals between the ages 30 and 50 having a typing speed greater than 50 words per minute. In addition, most of the participants had more than 3 years of experience with personal computers and Microsoft Word (see Tables 1 and 2).

Participants also answered a question regarding the types of training they had received and the percentages for each. The mean percentages are reported in Table 3 and show that the vast majority of respondents were self-taught.

Frequency of command usage. Figure 2 shows the mean reported percentage use of each command. The most important finding is that keyboard shortcuts were, in general, seldom used. Icon toolbars were used the most, followed by menus. The highest percentage use of keyboard shortcuts was for the editing com-

FIGURE 1 Example of a To copy material to the clipboard, specify the percent of time you use each of the following alternatives:

Click Edit, Click Copy

Alt, E, C

$\mathrm{Ctrl}+\mathrm{C}$

Click 酯

Right Click, Click Copy

Other (please specify method)

Total

question. 
Table 1: Demographic Data for the 251 Participants

\begin{tabular}{lrr}
\hline & $N$ & $\%$ \\
\hline $\begin{array}{l}\text { Typing speed (words per minute) } \\
<10\end{array}$ & 1 & \\
$11-20$ & 7 & 0.4 \\
$21-30$ & 34 & 2.8 \\
$31-40$ & 42 & 13.7 \\
$41-50$ & 41 & 16.9 \\
$>50$ & 123 & 16.5 \\
Job category & & 49.6 \\
Executive & 5 & \\
Manager & 26 & 2.0 \\
Professional & 172 & 10.4 \\
Clerical & 45 & 69.1 \\
Gender & & 18.1 \\
Female & 150 & \\
Male & 101 & 59.8 \\
Age & & 40.2 \\
20-29 & 35 & 13.9 \\
30-39 & 75 & 29.9 \\
$40-49$ & 84 & 33.5 \\
$50-59$ & 54 & 21.5 \\
$>60$ & 3 & 1.2 \\
\hline
\end{tabular}

Table 2: Participants' Experience With Personal Computers and Microsoft Word

\begin{tabular}{|c|c|c|}
\hline Question & $N$ & $\%$ \\
\hline \multicolumn{3}{|c|}{ How many years have you used a personal computer? } \\
\hline $1-2$ years & 8 & 3.2 \\
\hline $2-3$ & 12 & 4.8 \\
\hline More than 3 & 231 & 92.0 \\
\hline \multicolumn{3}{|c|}{ How many years have you used Microsoft Word? } \\
\hline$<1$ year & 33 & 13.1 \\
\hline $1-2$ & 41 & 16.3 \\
\hline $2-3$ & 46 & 18.3 \\
\hline$>3$ & 131 & 52.2 \\
\hline \multicolumn{3}{|c|}{ In a typical week, how much time do you spend using your personal computer? } \\
\hline$<1$ hour & 1 & 0.4 \\
\hline $1-5$ & 16 & 6.4 \\
\hline $5-10$ & 37 & 14.7 \\
\hline $10-15$ & 14 & 5.6 \\
\hline$>15$ & 183 & 72.9 \\
\hline \multicolumn{3}{|c|}{ In a typical week, how much time do you spend using Microsoft Word? } \\
\hline$<1$ hour & 33 & 13.3 \\
\hline $1-5$ & 81 & 32.5 \\
\hline $5-10$ & 62 & 24.9 \\
\hline $10-15$ & 31 & 12.4 \\
\hline$>15$ & 42 & 16.9 \\
\hline Missing & 2 & 0.8 \\
\hline
\end{tabular}


Table 3: Mean Percentage of Time Reported for Each of the Training Methods

\begin{tabular}{lrr}
\hline Method & $M$ & $S D$ \\
\hline Self-taught & 72.10 & 31.24 \\
Computer tutorials & 3.19 & 11.06 \\
Instructor-led training & 16.14 & 25.91 \\
Informal training from colleagues & 6.00 & 10.53 \\
Other & 0.58 & 5.45 \\
\hline
\end{tabular}

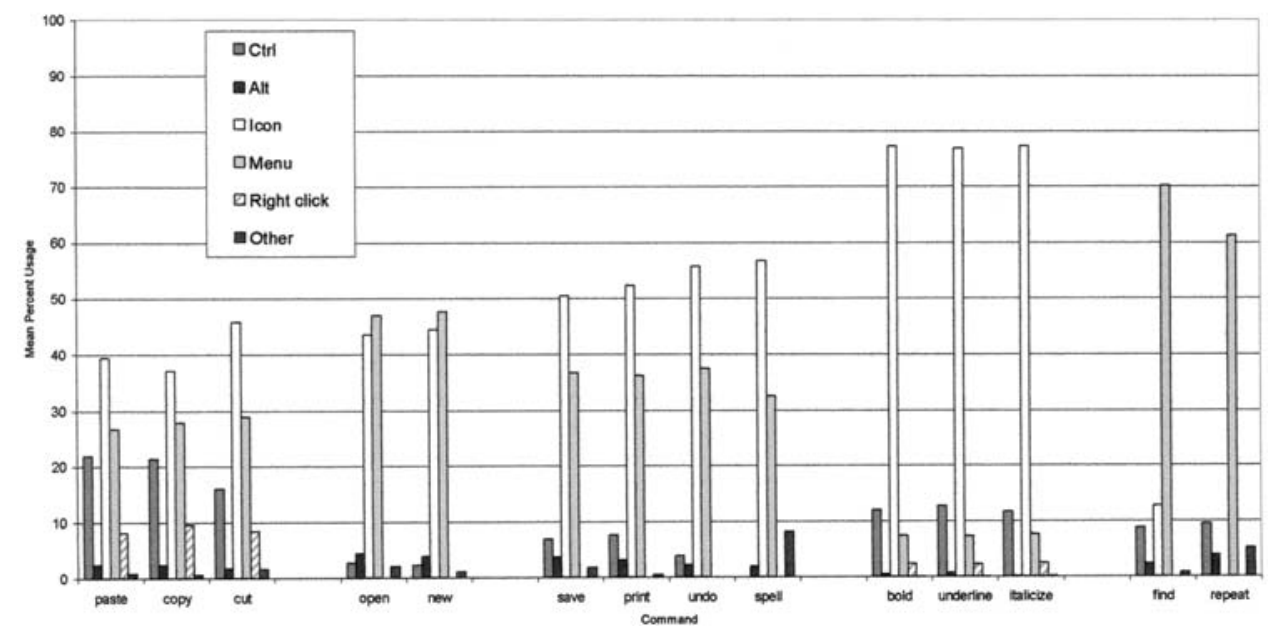

FIGURE 2 Mean reported frequency of method use as a function of command.

mands Cut, Copy, and Paste. However, even for these commands, the icon toolbar was used considerably more frequently than keyboard shortcuts.

Favorite method. To investigate individual differences in command methods, we tallied the percentage of users who favored each of the command methods. Two criteria were used to define the "favorite" method: a loose criterion and a strict criterion.

Loose Criterion: A method was considered a favorite method if its average percentage use was higher than any other method.

Strict Criterion: A method was considered a favorite method if its average percentage use was higher than any other and the average percentage use was higher than $60 \%$. If there were no methods with a percentage higher than $60 \%$, the participant was judged not to have a favorite method.

Table 4 shows the percentage of participants favoring each method. The analysis was done twice, once for all commands and once just for the commands Cut, Copy, 
Table 4: Percentage of Users who Favored a Method for all Commands and for Cut, Copy, and Paste Commands

\begin{tabular}{|c|c|c|c|c|}
\hline \multirow[b]{2}{*}{ Method } & \multicolumn{2}{|c|}{ All Commands } & \multicolumn{2}{|c|}{ Cut, Copy, and Paste } \\
\hline & Loose Criterion & Strict Criterion & Loose Criterion & Strict Criterion \\
\hline Alt-menu & 1.6 & 0.8 & 1.6 & 1.2 \\
\hline Keyboard shortcut & 6.4 & 1.6 & 19.1 & 13.9 \\
\hline Menu & 24.7 & 10.4 & 25.9 & 18.7 \\
\hline Icon & 65.0 & 35.9 & 43.4 & 30.7 \\
\hline Right click & 2.4 & 1.6 & 9.6 & 4.0 \\
\hline Other & - & - & 0.4 & 0.4 \\
\hline No favorite & - & 49.8 & - & 31.1 \\
\hline
\end{tabular}

and Paste. In the analysis considering all the commands, the method of keyboard shortcuts was the favorite method for only $6.37 \%$ of the users based on the loose criterion and for only $1.59 \%$ of the users based on the strict criterion. Considering just the Cut, Copy, and Paste commands, the method of keyboard shortcuts was the favorite method for $19.12 \%$ of the users based on the loose criterion and for $13.94 \%$ of the users based on the strict criterion.

To investigate individual differences in command use, a cluster analysis was performed utilizing Ward's minimum variance method using the SAS software package. In our judgment, the most interpretable result was obtained when the sample was divided into five clusters. The number of participants in each cluster and the description of each cluster are listed in Table 5. Participants in the "Icon Cluster" used the icon toolbar almost exclusively. Those in the "Menu Cluster," as the name implies, predominantly used the menu commands. The only exception was that they greatly preferred the icon toolbar to menu commands for the formatting options "Bold," "Underline," and "Italicize." The "Icon and Menu" cluster used both types of commands frequently. On some commands they used both methods about equally often whereas on others they preferred either the icon toolbar or menu commands. The "Some Shortcuts" cluster used the keyboard shortcuts the vast majority of the time for "Cut, Copy, and Paste" but rarely for other commands. Finally, the "More Shortcuts" cluster used keyboard shortcuts frequently but by no means exclusively for many commands. More detailed results from the cluster analysis are shown in Appendix A.

Table 5: Number of Participants in Each of the Five Clusters

\begin{tabular}{lc}
\hline Cluster & $N$ \\
\hline Icon & 45 \\
Menu & 60 \\
Icon and menu & 94 \\
Some keyboard shortcuts & 20 \\
More keyboard shortcuts & 32 \\
\hline
\end{tabular}


Relations among variables. Researchers suspected there would be a relation between typing speed and/or familiarity with Microsoft Word and the use of keyboard shortcuts. However, this was not the case. Familiarity with Microsoft Word correlated only .13 with use of keyboard shortcuts whereas typing speed correlated only .09. All of the eight correlations between measures of command use and the demographic variables were lower than .20.

Highly experienced users of Microsoft Word make little use of the efficient method of keyboard shortcuts, relying, instead, on the less efficient icon toolbar and menu methods. Moreover, greater experience with the software was not indicative of the use of more efficient methods. It was particularly striking that many participants used the extremely inefficient method of menus. Therefore, even though the graphical user interface appears to support the transition from less efficient to more efficient methods, most users fail to make this transition.

Before considering the broader implications of this result, we felt it was worthwhile to conduct a small experimental study to confirm that keyboard shortcuts are, in fact, faster than icon toolbars and menus. Study 2 provides this confirmation.

\section{STUDY 2}

This study compared the efficiency of three methods of issuing commands: keyboard shortcuts, icon toolbars, and menu commands. Also of interest was the effect of whether the user's hand was on the mouse or on the keyboard at the beginning of the task.

\subsection{Method}

Participants. Six MBA students from Rice University were paid to participate in the experiment.

Procedure. Participants were given a task in which they were to issue a command using a keyboard shortcut, an icon bar, or a menu. Four commands were used: Copy, Paste Open, and Save.

Participants each performed eight blocks of 24 trials. For three of the participants, Trials 1-12 of each block began with one hand on the keyboard and Trials 13-24 began with both hands on the keyboard. This order was reversed for the other three participants. Each set of 12 trials for a given hand condition consisted of 4 trials with the icon toolbar method, 4 trials with the menu method, and 4 trials with the keyboard shortcut method. The 4 trials for a given method were blocked and consisted of a Copy, a Paste, an Open and a Save command. The order of these blocks of four commands within the 12 trials was determined randomly for each set of 12 trials for each participant. The order of the three command methods was determined randomly for each participant for each set of 12 trials. 
Participants were given instructions and a few practice trials before beginning. The time it took to issue each command was recorded electronically.

\subsection{Results and Discussion}

The first four blocks of trials were considered practice and not included in the analysis. The mean response times as a function of method (icon toolbar, menu, and keyboard shortcut) and hand location are shown in Table 6 . The keyboard shortcut method was the fastest, the icon method was intermediate, and the menu method was the slowest, $F(2,10)=141.73, p<.01$. Beginning with one hand on the mouse led to slightly faster responding in the icon and menu conditions and slightly slower responding in the keyboard condition. The main effect of number of hands on the keyboard was significant, $F(1,5)=11.50, p=.019$, as was the Number of Hands $\times$ Method interaction, $F(2,10)=12.22, p<.01$. Table 6 also shows the mean response times separately for the four commands. The superiority of keyboard shortcuts is consistent across all of these commands. Table 7 shows confidence intervals on pairwise differences between mean response times for the three methods. In all cases, the lower limit on the interval was greater than .5 seconds. These findings

Table 6: Mean Response Times (in Sec) as a Function of Condition

\begin{tabular}{llcc}
\hline Command & Method & Both Hands on Keyboard & One Hand on Keyboard \\
\hline Overall & Keyboard & 1.362 & 1.508 \\
& Icon & 2.169 & 2.020 \\
Copy & Menu & 3.129 & 2.711 \\
& Keyboard & 1.332 & 1.366 \\
& Icon & 2.360 & 2.186 \\
Open & Menu & 3.170 & 2.685 \\
& Keyboard & 1.253 & 1.399 \\
& Icon & 2.058 & 1.702 \\
Paste & Menu & 2.743 & 2.561 \\
& Keyboard & 1.509 & 1.825 \\
& Icon & 1.954 & 2.205 \\
Save & Menu & 3.491 & 2.682 \\
& Keyboard & 1.354 & 1.443 \\
& Icon & 2.302 & 1.989 \\
& Menu & 3.112 & 2.915 \\
\hline
\end{tabular}

Table 7: Response Time Differences Between Conditions and Confidence Intervals on the Differences

\begin{tabular}{lccc}
\hline & Icon-Keyboard & Menu-Icon & Menu-Keyboard \\
\hline Mean difference & .659 & .825 & 1.484 \\
Standard deviation & .143 & .208 & .277 \\
Standard error & .058 & .085 & .113 \\
Lower 95\% limit & .509 & .607 & 1.193 \\
Upper 95\% limit & .809 & 1.043 & 1.775 \\
\hline
\end{tabular}


confirm that the keyboard shortcut method is substantially faster than the icon methods and that the icon method is substantially faster than the menu method.

A limitation of this study is that the editing commands were not issued during normal editing and therefore one should be careful about generalizing the details of the results to a normal editing task. However, the size and consistency of the effects as well as their congruence with everyday experience leaves little doubt that keyboard shortcuts are more efficient than the other methods.

\section{GENERAL DISCUSSION}

The findings from this study indicate that although the keyboard shortcut method is the most efficient, it is not frequently employed. It is particularly notable that even highly experienced users rarely employ keyboard shortcuts.

As discussed in the Introduction, the graphical user interface is easy to use and provides what appears to be strong support for the transition from easy-to-learn to easy-to-use methods. However, despite the apparent support, few users actually make the transition. As a result, graphical user interface users are not nearly as efficient as they could be. Naturally, the impact of interface efficiency on productivity depends on the proportion of the time the user is actually interacting with the interface rather than performing tasks such as gathering information and planning how to structure and present it. Doubling interface efficiency would not nearly double productivity. Nonetheless, it would not be unusual for a user to issue hundreds of commands a day, and the accumulation of small time savings could be considerable. Moreover, efficient methods of interacting with the interface may be less distracting than less efficient methods.

Results of this study are consistent with those of Bhavnani and John (1997), who found that participants with extensive experience using a CAD program often did not use it efficiently. What is striking about the results of this study, though, is that keyboard shortcuts are not used despite their being relatively easy to learn.

The results of the Bhavnani and John (1997) study, in combination with the results of the study reported here, suggest that experience alone is not sufficient for the adoption of efficient methods. Apparently, habitual patterns dominate performance and it is unusual for people to switch to new strategies. Specifically, people use a few functions they know well to accomplish their tasks and do not seek a new and possibly better method (Cornett, 1997). Even experienced users often utilize only a few functions to accomplish their task instead of searching for more efficient methods (Carroll \& Rosson, 1987).

Bhavani and John have completed several studies investigating the efficiency with which computer programs are used (Bhavnani \& John, 1998, 2000; Bhavnani, Reif, \& John, 2001). In their 2000 paper, they concluded that people often adopt inefficient methods either because they do not know about efficient methods or else choose not to use/learn them. We suspect that both factors contributed to the relative lack of use of keyboard shortcuts. Because $72 \%$ of the participants in this study indicated they were self-taught, these users may not have been fully aware of the keyboard shortcuts. However, we suspect that in many cases, users were aware 
that shortcuts existed, but did not expend the effort to learn them. This could be because they did not feel that the shortcuts would save much time or because they resisted making the initial investment of time necessary to learn the shortcuts.

As noted earlier, the icon method is the most popular method for issuing commands. Therefore, one way to help users learn the shortcut for a command would be to display the shortcut when the mouse pointer is placed on an icon. This is an option on Microsoft Word, but it is not the default. We suspect making this the default would be helpful but would not make a large difference. Recall that many users continue to use inefficient menu commands even though the keyboard shortcuts are shown with the menu commands.

Perhaps increased attention to efficiency during training would be effective. We are not suggesting that instructors devote much time to keyboard shortcuts in training sessions for novice users, for this would decrease the ease of learning associated with graphical interfaces. Instead, instructors could teach just two or three shortcuts and then provide users with a set of about 10 that should be learned over the course of several months. For example, trainees could be instructed to learn one additional shortcut per week.

Bhavnani et al. (2001) discussed the importance of generalizing efficient strategies across applications. The standardization of keyboard shortcuts across applications and even across platforms (Macintosh, Windows, and applications such as StarOffice in Linux) increases the payoff of learning a few shortcuts. Showing users the amount of time they could save across applications by using the keyboard shortcuts may increase the likelihood that they take the time to learn a few of them.

The basis of the large individual differences portrayed in the cluster analysis is unclear. These differences may be due to differences in motivation to work efficiently or they may be due to differences in cognitive ability and / or cognitive style. Because the source of these individual differences has implications for how to train users to work efficiently, further research exploring this issue appears merited.

Although keyboard shortcuts appear to be the most efficient method for issuing commands, they are not necessarily efficient for other types of interactions. For example, selecting a range of cells using keyboard commands can be very difficult (Napier, Lane, Batsell, \& Guadagno, 1989) and the use of a mouse is certainly more efficient.

It is important to keep in mind that this study did not measure actual computer usage. Instead, it relied on self-reports. Although there is no way to ascertain the accuracy of these reports, we suspect that, if anything, the self-reports overestimate the use of keyboard commands. We think it is unlikely that people who have learned efficient methods would report that they rely on easier to learn and less efficient methods.

\section{REFERENCES}

Bhavnani, S. K., \& John, B. E. (1997). From sufficient to efficient usage: An analysis of strategic knowledge. Retrieved April 3, 2002, from http://www.acm.org/sigs/sigchi/chi97/proceedings/paper/skb.htm 
Bhavnani, S. K., Reif, F., \& John, B. E. (2001) Beyond command knowledge: Identifying and teaching strategic knowledge for using complex computer applications. Proceedings of CHI' 01, 229-236.

Carroll, J. M., \& Rosson, M. B. (1987). Paradox of the active user. In J. M. Carroll (Ed.), Interface thought: Cognitive aspects of human-computer interaction (pp. 80-111). Cambridge, England: Bradley.

Cornett, L. L. (1997). Improving system knowledge and performance with proactive intelligent on-line help. Unpublished doctoral dissertation, Rice University, Houston, TX.

Davis, S., \& Bostrom, R. (1992). An experimental investigation of the roles of the computer interface and individual characteristics in the learning of computer systems. International Journal of Man-Machine Studies, 4, 143-172.

Galitz, W. O. (1996). The essential guide to user interface design: An introduction to GUI design principles and techniques. New York: Wiley.

Napier, A., Lane, D. M., Batsell, R., \& Guadagno, N. (1989). The impact of a natural language interface on ease of learning and productivity: A comparison of Lotus 1-2-3 and Lotus HAL. Communications of the ACM, 32, 1190-1198.

Rosson, M. B. (1984). Patterns of experience in text editing. Human Factors, 26, 463-475.

Temple, B., \& Sloane, Inc. (1990). The benefits of the graphical user interface. Lexington, MA: Microsoft and Zenith Data Systems.

$\mathrm{Wu}, \mathrm{J}$. (2000). Accommodating both experts and novices in one interface. Retrieved March 10, 2002 , from 
Appendix: Mean Percent Usage as a Function of Command and Cluster

\begin{tabular}{|c|c|c|c|c|c|}
\hline Five different clusters of users & Icon & Мепu & Icon/Menu & Some Shortcuts & More shortcuts \\
\hline $\mathrm{N}$ & 45 & 60 & 94 & 20 & 32 \\
\hline \multicolumn{6}{|l|}{ Cut, Copy, Paste } \\
\hline Control & 0.85 & 7.59 & 11.69 & 86.92 & 51.19 \\
\hline Alt & 0.00 & 0.86 & 1.38 & 0.10 & 11.56 \\
\hline Icon & 92.56 & 10.35 & 46.47 & 3.00 & 24.11 \\
\hline Menu & 5.33 & 76.59 & 19.95 & 5.43 & 5.68 \\
\hline Right Click & 1.23 & 2.69 & 18.48 & 1.13 & 6.47 \\
\hline Other & 0.00 & 0.22 & 1.70 & 3.42 & 1.04 \\
\hline \multicolumn{6}{|l|}{ Open, New } \\
\hline Control & 0.22 & 0.67 & 1.85 & 0.25 & 12.67 \\
\hline Alt & 0.02 & 1.11 & 3.20 & .10 & 19.50 \\
\hline Icon & 86.67 & 13.12 & 46.36 & 23.08 & 48.75 \\
\hline Menu & 12.81 & 84.53 & 45.18 & 71.58 & 18.14 \\
\hline Right Click & - & - & - & - & - \\
\hline Other & 0.06 & 0.58 & 2.73 & 3.75 & 0.94 \\
\hline \multicolumn{6}{|l|}{ Bold, Underline, Italicize } \\
\hline Keyboard Shortcut & 2.00 & 5.56 & 4.89 & 0.00 & 67.32 \\
\hline Alt & 0.00 & 0.44 & 0.94 & 0.00 & 0.00 \\
\hline Icon & 96.81 & 71.22 & 85.12 & 93.00 & 27.41 \\
\hline Menu & 1.19 & 21.03 & 4.14 & 7.00 & 0.95 \\
\hline Right Click & 0.00 & 1.14 & 4.09 & 0.00 & 4.06 \\
\hline Other & 0.00 & 0.00 & 0.11 & 0.00 & 0.26 \\
\hline \multicolumn{6}{|l|}{ Save, Print, Undo, Spell } \\
\hline Keyboard Shortcut & 1.14 & 3.48 & 3.81 & 7.42 & 24.05 \\
\hline Alt & 0.02 & 0.65 & 1.74 & 1.58 & 14.26 \\
\hline Icon & 87.90 & 28.16 & 59.14 & 48.41 & 41.55 \\
\hline Menu & 10.54 & 65.99 & 33.37 & 38.26 & 19.87 \\
\hline Right Click & - & - & - & - & - \\
\hline Other & 0.53 & 1.73 & 1.56 & 4.25 & 5.03 \\
\hline \multicolumn{6}{|l|}{ Find, Repeat } \\
\hline Keyboard Shortcut & 7.36 & 2.79 & 5.35 & 23.50 & 25.55 \\
\hline Alt & 2.50 & 0.79 & 2.34 & 2.18 & 10.94 \\
\hline Icon & 41.33 & 4.75 & 9.10 & 3.50 & 3.28 \\
\hline Menu & 62.82 & 79.88 & 68.09 & 52.83 & 44.06 \\
\hline Right Click & - & - & - & - & - \\
\hline Other & 1.09 & 0.83 & 2.29 & 14.75 & 3.75 \\
\hline
\end{tabular}

